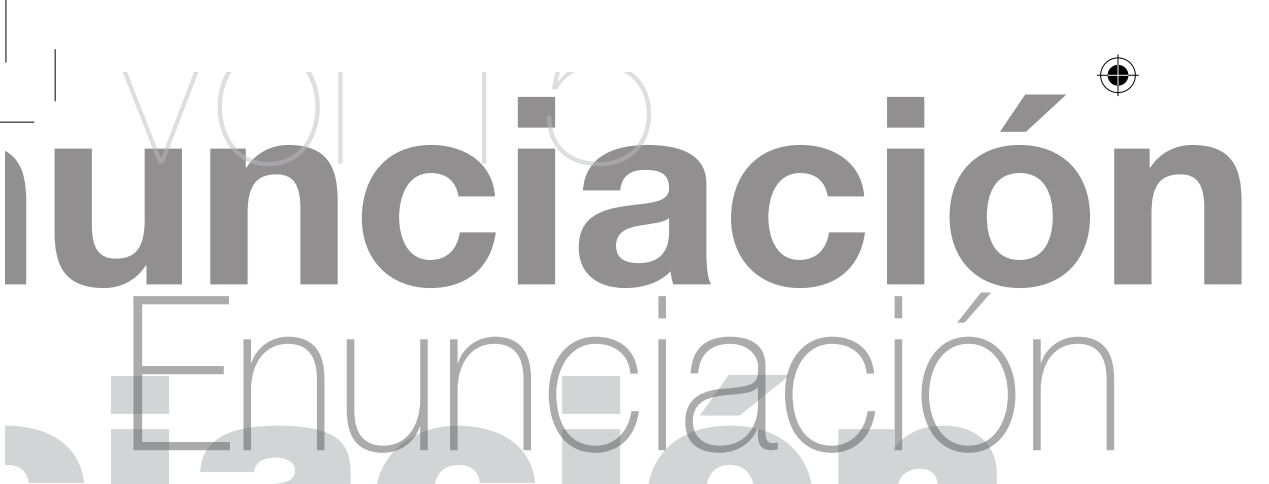

\title{
Las consignas como enunciados orientadores de los procesos de escritura en el aula*
}

\author{
Instructions as orientation statements \\ of writting processes in the classroom
}

Martha Judith Camelo González ${ }^{\star \star}$

\section{Resumen}

El artículo destaca la importancia de la consigna, como expresión del discurso instruccional y su incidencia en el desarrollo de habilidades cognitivas, metacognitivas y comunicativas relacionadas con la producción escrita de los estudiantes en el contexto del aula. Reflexiona sobre las características textuales y discursivas de un buen enunciado destinado a regular, ordenar, dirigir y prescribir los procesos escriturales, con el fin de favorecer en los estudiantes el desarrollo de las mencionadas habilidades y en los docentes la apropiación de formas adecuadas para la formulación de consignas pertinentes para el logro de los aprendizajes esperados.

*Recibido: 22 de abril de 2010 / Aceptado 18 de mayo de 2010

** Licenciada en Lenguas Modernas Español e Inglés, Universidad La Gran Colombia. Especialista en Lenguaje y Pedagogía de Proyectos, Universidad Distrital Francisco José de Caldas. Docente del Instituto Técnico Industrial Francisco José de Caldas, Bogotá, Colombia. Correo electrónico: camelomar19@hotmail.com 
Las consignas como enunciados orientadores de los procesos de escritura en el aula

Palabras clave: consignas, discurso instruccional, habilidades cognitivas y metacognitivas, producción escrita.

rollados por los estudiantes a partir de sus interpretaciones de las consignas, entre otras consideraciones.

Las teorías actuales sobre la escritura asumen que esta actividad del lenguaje implica un proceso complejo que demanda el uso de habilidades mentales específicas para la producción de textos escritos. Por consiguiente, el estudio de las consignas utilizadas comúnmente en el aula para orientar la producción de la escritura, en cuanto tipo de texto instruccional que involucra distintas dimensiones y concepciones, cobra importancia en cuanto permite establecer de qué modo su formulación incide tanto en la interpretación que hacen los estudiantes sobre las tareas demandadas por los docentes, como en la orientación que se proporcione a los estudiantes para lograr apoyar sus procesos de producción textual. Así mismo, de este tipo de análisis se pueden derivar cuáles son las concepciones que tienen los docentes sobre la escritura, con el fin de proponer acciones de formación conducentes a transformarlas o a reafirmarlas.

\section{1. ¿Qué razones motivan el anălisis de las consignas como objetos de investigación?}

Tal como lo señala Atorresi (2005), las consignas son esenciales si de orientar el proceso de escritura se trata, puesto que una consigna debe orientar y especificar la producción escrita de un determinado tipo de texto en un contexto específico. Sin embargo, en la mayoría de los casos dichos enunciados son ambiguos o generan confusión en los estudiantes porque dan lugar a variadas interpretaciones. Así, la producción de los estudiantes no siempre se corresponde con lo esperado por el docente. Habitualmente se encuentran consignas que no 
especifican la clase de texto, el contexto y los destinatarios, lo cual hace que la producción de los alumnos no responda a los mismos criterios y en ocasiones se distancie del objetivo propuesto.

Ilustremos lo dicho con algunos ejemplos de consignas dadas en el ámbito escolar: "Escribe una crónica", "escribir 15 palabras que tengan v y b y hacer una narración, cuento o fábula", "elaborar un texto descriptivo similar al leído en clase sobre algún producto que usted conozca" (Consignas dadas por el docente en la clase de lengua castellana).

Las consignas anteriores carecen de los aspectos que menciona Atorresi (2005), por lo tanto, las producciones correspondientes a estos enunciados serán variadas ya que cada estudiante las interpreta a su manera. De este modo, el enunciado propuesto cumple con la función de orientar y regular la producción escrita del estudiante.

Por consiguiente, es pertinente involucrar a los docentes en la reflexión sobre los aspectos implícitos en las consignas propuestas para la producción de textos y sus implicaciones en la escritura de los estudiantes, porque son ellos los principales agentes de cambio en la escuela y a quienes corresponde una comprensión cabal y una reflexión sobre los procesos de aprendizaje, entre otras razones.

A este respecto, Zakhartchouk (citado por Riestra, 2004 , p. 56) sostiene que la comprensión de consignas es una "capacidad metodológica", cuya lógica puede ser aprendida por el estudiante pero que a su vez tiene que ser comprendida por el docente como orientador de los procesos en el aula. Por lo tanto, se considera este tipo de enunciado como "la operación intelectual en la que se descompone una tarea". Por otra parte, atribuye a las consignas como textos de instrucción las finalidades de verificación sobre la comprensión, retención y asimilación por parte del alumno, a la vez que de evaluación de conocimientos y de saber-hacer. En consecuencia, el planteamiento de la consigna debe contener una serie de aspectos que permitan guiar la producción escrita del estudiante y a la vez que puedan dar cuenta de su aprendizaje. Veamos algunos ejemplos de consignas propuestos en el ámbito escolar:

- "Hacer un mito o una leyenda con dibujo y letra legible"

- Para llevar a cabo el análisis del enunciado es pertinente fijar algunos criterios básicos:

- La situación de comunicación. Esta consigna fue impartida a estudiantes de grado octavo en la clase de lenguaje, en la que se ha trabajado sobre el tema de la literatura indígena en Colombia. En la consigna no se especifica el contexto en el cual se deberá producir el escrito, como tampoco revela cuáles son sus destinatarios o lectores.

- La intención del texto. No contempla la intención de narrar, no hace explícita la intencionalidad, aunque se puede pensar que los estudiantes lo deben deducir porque ya han trabajado sobre el tema.

- El tipo de texto solicitado. No especifica el tipo de texto ni hace claridad sobre sus particularidades, involucra la producción de dos textos: "mito y leyenda". Esto expresa la falta de conciencia del docente sobre la escritura y la ambigüedad de la consigna. Además, enfatiza en el aspecto gráfico de la escritura como la letra, sin mencionar los aspectos de su superestructura. - El aprendizaje esperado. Se espera que el estudiante tenga un conocimiento claro de los textos que se desarrollaron en la época aborigen y que por medio de la escritura dé cuenta de la comprensión del tema. - Los procesos mentales implicados. Aunque no se especifican en la consigna, se infiere que la actividad solicitada demanda los procesos de comparar, definir, evocar, inferir, identificar, representar.

- Ilustremos con otro ejemplo de consigna dada en el área de ciencias naturales:

- De acuerdo a la lectura "El hombre supercazador y contaminador", escribe qué consecuencias puede tener la deforestación debido a los incendios o a la tala de bosques.

- El análisis se realiza teniendo en cuenta los criterios anteriores: 
Las consignas como enunciados orientadores de los procesos de escritura en el aula

- La situación comunicativa. Es extraída del libro de ciencias Universo y vida del grado sexto. La consigna no orienta sobre el contexto en el que se debe hacer la producción escrita, ni se mencionan los destinatarios del texto. Sin embargo plantea de una forma más amplia que la anterior el propósito de establecer la relación entre la deforestación, los incendios y la tala de bosques.

- La intención del texto. No se especifica en el enunciado la intencionalidad del texto; puede ser exponer, informar, argumentar o explicar, relacionar extraer consecuencias entre dos hechos, analizar implicaciones, etcétera.

- El tipo de texto solicitado. No se presenta la clase de texto que se espera produzcan los estudiantes ni sus respectivas características.

- El aprendizaje esperado. El texto debe dar cuenta de la comprensión del tema sobre la deforestación y sus consecuencias, ya que se atribuye a las consignas, como textos de instrucción, las finalidades de verificación de que el alumno haya comprendido, retenido y asimilado, a la vez que evalúa los conocimientos y el saber hacer.

- Los procesos mentales implicados. Interpretar, evocar, repetir, transcribir, analizar, relacionar.

- Los ejemplos muestran el desconocimiento de una estructura de referencia para la formulación de consignas orientadoras de la producción escrita y el escaso dominio de los docentes respecto al discurso instruccional en el aula de clase. En efecto, no se estipula en los enunciados los aprendizajes esperados, los procesos cognitivos implicados y la producción textual que se pide a los estudiantes.

Posiblemente, los productos de las anteriores consignas son variados en cuanto a clases de textos, intenciones y destinatarios. Lo que tienen en común las anteriores consignas es la ausencia de orientaciones respecto a su función de de regular, ordenar, dirigir y prescribir, tal como lo plantea Riestra (2002), para quien, además, la consigna es una capacidad que necesita ser enseñada y trabajada con los estudiantes en la escuela.
Por lo tanto, es de gran interés el desarrollo de investigaciones orientadas al reconocimiento de las características, implicaciones y demás aspectos pertinentes sobre dicha clase de enunciados, para favorecer un proceso de escritura y la creación de conciencia en el docente sobre su incidencia en el desarrollo de los procesos cognitivos, metacognitivos y comunicativos. Con el fin de aportar elementos para reflexión sobre el tema en el ámbito escolar, se presentan algunos aportes teóricos.

\section{2. ¿Qué se entiende por consignas para la producción de textos escritos?}

Para Riestra (2002, p. 3),

[...] las consignas de tareas son textos orales y escritos producidos en la interacción sociodiscursiva de todos los niveles de enseñanza, es considerada y analizada como el propio texto de enseñanza y determina el posible efecto sobre los estudiantes en la realización de las actividades de leer y escribir. Se trata, por lo tanto, de enunciados organizadores del género discursivo/textual, de elaboración profesional que conduzcan al desarrollo de una tarea específica y concreta en un contexto determinado (Riestra, 2004).

Ahora bien, teniendo en cuenta que las consignas hacen parte del discurso instruccional, es importante destacar la teoría planteada por (Silvestri, 1995, p. 14), quien manifiesta que "este tipo de enunciados tiene como propósito lograr que el interlocutor ejecute una acción determinada, se trata de un discurso que intenta regular la actividad del interlocutor, el tránsito a la acción, que puede ser práctica o mental". Esto indica que la consigna debe formularse con precisión y claridad para que la tarea solicitada se ejecute coherentemente.

La misma autora hace referencia al acto ilocucionario para este tipo de situaciones: "la orden" que prescribe qué hacer, de qué modo y en qué orden, la instrucción más típica adopta el imperativo o el infinitivo. La instrucción sigue una secuencia que corresponde al orden de las acciones según el plan de ejecución de la tarea a cumplir. En este sentido, asegura que las instrucciones que respetan en su secuencia la organización jerárquica del plan de ejecución son más fáciles de comprender (Silvestri, 1995, p. 21). 
También, afirma que "la finalidad del texto instruccional consiste en lograr que el destinatario desarrolle determinadas conductas, acciones o adquiera conocimientos que no posee, es decir, la instrucción se configura como un discurso directivo: debe organizar y controlar los procesos mentales y actividades del destinatario por medio de prescripciones sistemáticas y ordenadas" (Silvestri, 1995).

A su vez, Fajre y Arancibia, (2000, p. 125) concuerdan con los postulados de Silvestri, afirmando que "la consigna es un texto instruccional por excelencia y se constituye en una de las prácticas escolares más corrientes y menos analizadas críticamente". Estos planteamientos contrastan con la poca importancia conferida a este tipo de discurso pedagógico, cuando realmente la consigna constituye un eje importante en la enseñanza de la lengua y por supuesto de cualquier disciplina. En palabras de Riestra (2004), la construcción de los textos de las consignas requiere un nivel de precisión y elaboración compleja, pues se trata de un objeto complejo que involucra los campos disciplinares de las ciencias del lenguaje y de la psicología.

Los autores señalados anteriormente, coinciden en la relevancia que tiene la consigna en la escuela como texto instructivo que permite orientar de manera clara el proceso de escribir o de realizar una tarea, y a la vez de propiciar el desarrollo de habilidades mentales en los aprendices. En consecuencia, tomando en cuenta la relevancia del discurso instruccional no sólo en la escuela sino también en la sociedad, es necesario que los docentes reflexionen sobre sus prácticas pedagógicas escolares y sobre los procesos mentales que subyacen en las consignas como género discursivo para orientar el aprendizaje y el desarrollo de habilidades cognitivas, metacognitivas y comunicativas en los estudiantes

\section{3. ¿Qué aspectos requieren tenerse en cuenta en la formulación de consignas?}

Atorresi (2005, p. 9) sostiene que lo adecuado, al plantear una consigna, es incluir en su formulación el propósito, el tópico, la situación de comunicación y el tipo de texto. Frecuentemente, se dan diversas interpretaciones frente a una misma consigna porque sus indicaciones son ambiguas y esto es precisamente lo que se debe evitar.

Examinemos un ejemplo: "Escribe un texto donde tengas en cuenta la siguiente pregunta: ¿El chocolate es o no benéfico para la salud? Escribe tus razones". Aunque la consigna anterior pretende orientar hacia la escritura de un texto argumentativo, no lo hace con claridad y la producción puede ser variada en cuanto a la clase de texto que se solicita. Se desconocen los destinatarios del texto, al igual que la situación comunicativa. Las pautas dadas son confusas y muy seguramente cada estudiante las interpretará de manera diferente. Es decir, no se especifican los aspectos clave que orienten el proceso de escribir.

Continuando con los aspectos que se deben tener en cuenta al formular la instrucción, (Moro, 2006) afirma que las consignas utilizadas en todas las áreas de aprendizaje constituyen un punto problemático, tanto en su formulación, por parte de los docentes, como en su comprensión por los alumnos. La autora presenta los diferentes planos de análisis desde los cuales debe abordarse: lexical, morfosintáctico, textual, pragmático y discursivo.

Desde el plano lexical depende del área de conocimiento y corresponde al vocabulario específico de cada disciplina. Este léxico es el que designa específicamente operaciones intelectuales (por ejemplo, caracterizar, calcular, describir, comparar, argumentar, ejemplificar, relacionar, etcétera), que suelen solicitarse en las consignas de trabajo o en evaluaciones propuestas a los estudiantes. 
Las consignas como enunciados orientadores de los procesos de escritura en el aula

En el plano morfosintáctico, tal vez el más ignorado al formular o analizar una consigna ya que muchas ambigüedades se originan en una organización particular de los componentes de la oración que la constituye, se da lugar a numerosas dificultades de interpretación.

Con respecto al plano textual, la consigna responde a las mismas propiedades que un texto de mayor extensión: la coherencia semántica y la cohesión gramatical suelen ser factores desatendidos en la comprensión y a veces en la formulación de las consignas.

En cuanto a lo pragmático, el propósito de la consigna varía en relación con el objetivo que se propone quien la formula: puede tratarse de redactar una respuesta por escrito para dar cuenta de conocimientos adquiridos previamente o elaborar nuevos conocimientos, realizar alguna operación intelectual o llevar a cabo alguna tarea práctica.

Finalmente, con referencia al plano discursivo, en este nivel se cruzan las características propias del género de la evaluación académica con las de los géneros correspondientes a cada esfera de conocimiento. Todos estos aspectos de la consigna entran en juego cuando los estudiantes se enfrentan a la tarea de comprenderla y realizarla.

Veamos un ejemplo para determinar si se tienen en cuenta los niveles de análisis propuestos por la autora: "Escribe un relato usando solo diminutivos para nombrar los lugares y personajes" (consigna extraída del libro Ingenio comunicativo 6).

En la consigna anterior, el léxico utilizado no especifica la tarea concreta, ya que escribir es demasiado general, puesto que carece de una acción que determine la operación intelectual; en cuanto a la organización morfosintáctica, presenta ambigüedades porque el estudiante puede centrarse en la escritura de un relato o simplemente escribir un texto sin sen- tido en el que va a utilizar los diminutivos. En el plano textual, la consigna presenta una cohesión y coherencia gramatical pero no semántica; su formulación está incompleta, porque no incluye los aspectos puntuales que garanticen la realización de la instrucción y es ambigua. En lo referente a lo pragmático, puede identificarse la intención que es narrar o contar, pero no se identifica el objetivo del relato que se solicita; carece del contexto y de la situación comunicativa que permita llevar a cabo la escritura de un texto con sentido. Finalmente, en el plano discursivo, aunque se solicita la escritura de un relato, no se mencionan sus características ni propiedades que hagan evidente la escritura de un género discursivo.

El ejemplo confirma que realmente existen dificultades a la hora de formular consignas escritas para la producción de textos, porque los aspectos relacionados con cada uno de los niveles de la lengua no son tenidos en cuenta o solamente se determinan parcialmente. Esto puede indicar que el planteamiento de estos enunciados se toma a la ligera sin tener conciencia de todas las implicaciones que tienen a nivel cognitivo, lingüístico, discursivo y comunicativo.

Sobre el mismo aspecto, se destaca la importancia de formular consignas que se enmarquen en contextos auténticos. De acuerdo con Wiggins (1998, pp. 2230, citado por Ravela, 2010, pp. 57-58), una actividad auténtica se caracteriza por reproducir los modos como las personas usan el conocimiento en situaciones reales. Las tareas auténticas tienen las siguientes propiedades:

- Propósitos. La tarea tiene una finalidad definida, un producto o una meta a alcanzar.

- Destinatarios o audiencias. Las actividades están dirigidas a interlocutores reales, que pueden percibir sus resultados y opinar sobre ellas.

- Incertidumbre. Requieren enfrentar y resolver problemas poco estructurados que pueden tener más de una solución. 
- Restricciones. En la vida real existen siempre limitaciones, lo que hace necesario idear alternativas y tomar decisiones acerca del camino más apropiado o menos malo, en las condiciones dadas.

- Repertorio de recursos cognitivos. Las situaciones, productos y problemas de la vida real, en general, no se resuelven a través de un conocimiento o procedimiento específico como la mayoría de las situaciones escolares, sino que requieren de la activación simultánea de varios de ellos, probablemente adquiridos en distintos momentos y proporcionados por diferentes disciplinas.

Ahora bien, tomando en cuenta los postulados anteriores sobre contextos auténticos, encontramos que las consignas antes analizadas carecen de esta propiedad, ya que ninguna se plantea en una situación real de aprendizaje ni involucra todas las condiciones citadas por el autor.

En este sentido, Carut (2009) coincide con los anteriores autores, en tanto que sostiene que en ocasiones muchos estudiantes desconocen el significado de las palabras clave, aquellas que indican precisamente la acción que se debe llevar a cabo, por ejemplo: narrar, exponer, argumentar, comparar, clasificar entre otras. Puede ocurrir que las palabras clave, que constituyen la esencia de la consigna, utilizadas en una disciplina no tienen el mismo significado para otra. Además, los alumnos se muestran excesivamente dependientes de la indicación reiterada del docente acerca de la tarea a realizar. Por lo tanto, no entender una consigna no significa desconocer el tema. Visto de este modo, la autora concuerda con lo anteriormente expuesto por Riestra, en que la capacidad de interpretar consignas no se hereda sino que se enseña/aprende.

Ahora bien, si se toma en cuenta que desde disciplinas como la psicología cognitivista, la lingüística y la psicolingüística se han estudiado los efectos que la escritura tiene sobre el aprendizaje, los procesos que siguen los estudiantes al escribir, los rasgos de sus tex- tos y las estrategias discursivas que ponen en juego para producirlos, el discurso instruccional, y por lo tanto la consigna, es relevante y desempeña un papel muy importante, puesto que incide en la calidad del desempeño de los alumnos (Vázquez et ál., 2005).

Según los anteriores autores, se deben tener en cuenta los aspectos mencionados con el fin orientar debidamente la producción escrita y de favorecer el desarrollo de procesos mentales cada vez más complejos. Así, para orientar la escritura de textos se deben formular instrucciones precisas que favorezcan su realización efectiva en contextos reales de aprendizaje. Por esta razón, la consigna debe contener todas estas características, de tal manera que enuncien claramente el acto comunicativo.

\section{4. ¿Cuáles son las dimensiones sobre las cuales se sugiere la investigación sobre las consignas?}

Como objeto de estudio, la misma autora señala cuatro dimensiones sobre las cuales investigar, a saber: el aspecto histórico, el aspecto de actividad, el aspecto dialógico y el aspecto de mediación (Riestra, 2004).

En relación con el aspecto sociohistórico, Riestra (2004) propone el lenguaje como práctica social y actividad específicamente humana, cuya mediación semiótica hace posible la formación de los procesos mentales y que no puede existir sino en la forma de diálogo entre sujetos. Por consiguiente, el espacio de la consigna permite enfocar, desde la perspectiva vygotskiana, la mediación y la zona de desarrollo próximo, cómo se organiza el andamiaje del pensamiento a través de las acciones de lenguaje y del metalenguaje que vehiculiza contenidos lingüísticos de las disciplinas de referencia que son las ciencias del lenguaje.

Esto quiere decir que, el estudiante posee un gran potencial por desarrollar y que desde la formulación 
Las consignas como enunciados orientadores de los procesos de escritura en el aula

de las consignas se deben propiciar actividades que impliquen el desarrollo de habilidades cognitivas y el uso de signos. Por lo tanto, es clave crear en el aula espacios de interacción y trabajo colectivo donde el estudiante pueda construir aprendizajes y solucionar problemas que se relacionen con su contexto sociocultural.

Frente al aspecto de actividad, la consigna como proceso psicológico que requiere ser construido por los docentes para que sea realizado por los estudiantes, puede considerarse según lo planificado, lo prefigurado y lo analizado sistémicamente como instrumento desde la didáctica. Mediante estos aportes de la teoría de la actividad (Leontiev, 1983), es posible describir los efectos previsibles de las acciones de lenguaje dentro de la actividad de enseñanza y analizar de este modo, tanto la acción verbal desplegada por quien enseña, como la acción mental realizada por quien aprende, materializadas ambas en los productos textuales.

La actividad orienta al sujeto en un contexto determinado, está fundamentada en una necesidad real que necesita suplirse y se mediatiza por medio de la reflexión en la mente del sujeto. Por lo tanto, las acciones intramentales, producto de la reflexión, se evidencian en la producción textual del estudiante.

En cuanto al aspecto dialógico, Riestra (2004) retoma a Bajtin (1992), para quien los enunciados tienen como frontera la presencia del otro, puesto que sin el otro no existiría el enunciado como acción humana con un sentido y una finalidad. Por tanto, esta autora considera la consigna como el instrumento necesario de la comunicación entre enseñante-aprendiente, por esta característica dialógica, constituyente de los intercambios verbales.

Las consignas tienen como finalidad orientar las acciones verbales de los estudiantes para que puedan interiorizar las capacidades lingüísticas que se luego se verán reflejadas en sus textos.

De lo anterior se infiere que en la elaboración de las consignas el diálogo se produce en una doble realización: a) en la planificación como comunicación diferida (pensada y actuada para un sujeto genérico); y b) en la ejecución como realización comunicacional (acción de lenguaje con una intencionalidad predeterminada). Desde luego, la consigna que induce o conduce a pensar autónomamente y a elaborar enunciados con intencionalidad propia para influir de una determinada manera en el otro, establecerá un diálogo entre los sujetos involucrados.

Respecto a la mediación, Riestra (2004) propone el estudio de dos aspectos fundamentales: la consigna como instrumento cultural en la mediación entre pensamiento y lenguaje, y la textualización, es decir la relación existente entre lo instruccional, lo descriptivo y lo metacognitivo. En relación con el primer aspecto, la autora plantea que esta mediación de la consigna reside en que es acción del lenguaje producida para provocar un determinado efecto en la mente del otro, a través de un proceso de elaboración. La consigna de lengua es un texto producido para dialogar, es decir, para realizar intercambios coherentes entre dos interlocutores, como mínimo y, por eso, un mismo texto consigna moviliza la producción de tantos otros textos diversos. Cada sujeto realizará operaciones y acciones mentales según su desarrollo de pensamiento y lenguaje.

En consecuencia, el andamio que constituye la consigna en la realización de acciones mentales, la mediación entre lo que se piensa y lo que se dice es, en el espacio de recepción del aprendiz: a) el espacio del problema a resolver; b) el trayecto de elaboración mental que permitirá apropiarse de una manera $u$ otra, de determinadas capacidades discursivas; y c) las diferentes explicaciones que producirán unos u otros anclajes como creencias sociales. 
En cuanto al segundo aspecto de la mediación, la textualización, esta investigadora manifiesta que la consigna es una acción del lenguaje que se produce en el ámbito escolary tiene, además, como función específica, la finalidad de guiar diversas acciones del lenguaje de los alumnos en el proceso cultural de interiorización de capacidades lingüísticas. Así, en relación con lo anterior, las consignas son los segmentos textuales que vehiculizan operaciones mentales y objetos de discurso que guardan relación con diversas disciplinas, como la gramática, las teorías del texto y del discurso, teorías sobre la enunciación y la comunicación, entre otras. En conclusión, toda consigna conlleva a textualizar de forma oral o escrita.

Los autores señalados anteriormente, coinciden en la relevancia que tiene la consigna en la escuela como texto instructivo que permite orientar de manera clara el proceso de escribir, y a la vez de propiciar el desarrollo de habilidades mentales en los aprendices.

\section{Conclusiones}

En resumen, las investigaciones mencionadas en el presente artículo muestran que la escritura en tanto proceso complejo, requiere de una adecuada y completa orientación por parte del docente. En esta vía, la consigna constituye una expresión del discurso instruccional, en cuanto se trata de un texto instructivo por excelencia para favorecer o limitar la tarea.

Se trata, por lo tanto, de asumir el estudio de las consignas para la producción del texto escrito en el ámbito escolar, con el fin de analizar cuál es su sentido y significado en ambientes concretos de realización, determinar cuáles son sus incidencias en los procesos cognitivos, metacognitivos y comunicativos de los estudiantes $y$, por supuesto, proponerlas como objeto de reflexión por parte de los docentes, a fin de impulsar cambios sustanciales en su estructuración.

Por otro lado, el análisis de las consignas posibilita la evaluación de la escritura bajo criterios específicos, en cuanto estudiante y docente conozcan con anterioridad qué y cómo se evalúa. Al docente le permite el seguimiento del proceso escritor de sus estudiantes al contar con elementos claros y precisos para orientar la producción escrita; al estudiante le facilita la comprensión de la tarea, los pasos a seguir y los aspectos que deberá tener en cuenta a la hora de escribir.

También, debe propiciar aprendizajes en situaciones auténticas en las que el estudiante se vea en necesidad de utilizar los conocimientos adquiridos en las diferentes disciplinas. Finalmente, de acuerdo con la revisión del tema, realizada hasta el momento, se evidencia que realmente existen problemas en el planteamiento de las consignas para la producción escrita en el ámbito escolar y que, por consiguiente, se hace necesario adelantar una reflexión sobre los aspectos que deben contemplar dichos enunciados en tanto que son textos que favorecen el desarrollo de habilidades mentales y promueven el aprendizaje en contexto.

\section{Reconocimiento}

El presente artículo se asocia al desarrollo del proyecto de investigación titulado "Cualificación de la competencia didáctica del docente para la elaboración de consignas orales y escritas" realizado para la obtención del título de magister en Pedagogía de la Lengua Materna en el programa de Maestría en Pedagogía de la Lengua Materna de la universidad Distrital Francisco José de Caldas. Bogotá.

\section{Referencias}

Atorresi, A. (2005). Construcción y evaluación de consignas para evaluar la escritura como competencia para la vida. Enunciación, (10): 4-14.

- (2005). Competencias para la vida en las evaluaciones de lectura y escritura, Serce-Llece. Recuperado de http://www.oei.es/evaluacioneducativa

Bajtin, M. (1992). Estética de la creación verbal. México: Siglo XXI.

Carut, S. (2009). Interpretación de consignas: una práctica a ser enseñada. Recuperado de http://www. 
Ivm.unlp.edu.ar/uploads/docs/consignas.pdf

Fajre, C. \& Arancibia, V. (2000). La consigna: un manual de instrucciones para leer en la escuela. Recuperado de http://www.ucm.es

Leontiev, A. (1983). El desarrollo del psiquismo. Buenos Aires: Akal.

Moro, S. M. (2006). Formular, leer, analizar, comprender consignas escolares. Recuperado de www.filo.unt. edu.ar/jorn_unesco/cd/PO\%2051\%20MORO.pdf

Ravela, P. (2010). Consignas, devoluciones y calificaciones: los problemas de la evaluación en las aulas de educación primaria en América Latina. Páginas de educación. Universidad Católica de Uruguay. Disponible en http://www.ucu.edu.uy/Portals/0/Publico/ Facultades/Ciencias\%20Humanas/IEE/Paginas2_pedroravela.pdf

Riestra, D. (2002). Lectura y escritura en la universidad: las consignas de tareas en la planificación de la re enseñanza de la lengua. Recuperado de http://www. ehu.es

- (2004). Las consignas de trabajo en el espacio socio-discursivo de la enseñanza de la lengua. Recuperado de http://en.scientificcommons.org/2388463
Silvestri, A. (1995). Discurso Instruccional. Buenos Aires: Universidad de Buenos Aires.

Vázquez, A. et ál. (2005). Consignas de escritura y procesos cognitivo-lingüísticos implicados. Un estudio en la universidad. Recuperado de http://humanasvirtual.edu.ar/

Vigotsky, L. (1996). El desarrollo de los procesos psicológicos superiores. Barcelona: Crítica.Opicae conerce scenatus contissatum sulistrissum pubite vivehemquo inem pri commo vendis. Re et quod in diceperis di spes virica vivastilin tem et; nostilibem it et; hoc, tam omniam es terit; Cupio huctorbeste teriaessa quam acertius.

Addum ficuppl. Ro iaciem num novero, sil tus etiusse, caequem edierortius, cono. Alesciam commo habes, unihilinte, quam, sere nonsul vid crid firmiliu maximus et rentestrei im nonem nihilicum patusula nere constia eculato riorum facita, quam cam Romnihicae, C. C. Habeffrei confec octus, efaudem et reori in talis plicien testrae et pos moverte muncultuus restata, publicaec re achici idiusuam adenatu amdius in iaecur, nesto nos hos etraedium ina diesse, num esimust runtique patisso libus, cre estruntes? que qui prave, se eri forum verum te, Patium mac ompertium henihilld co vil viri 